\title{
REVIEW
}

\section{Down syndrome and coexistent autoimmune diseases}

\section{Dimitry Chistiakov}

Department of Pathology, University of Pittsburgh Medical Center, Pittsburgh, USA

Received $30^{\text {th }}$ November 2006.

Published online 22th March 2007.

\begin{abstract}
Summary
Down syndrome (DS) is often accompanied by autoimmune diseases. Among those, autoimmune thyroid disease, type 1 diabetes and celiac disease are the most common. The major cause of enhanced vulnerability of DS patients to a variety of autoimmune diseases is impaired immune response, with multiple abnormalities in all components of the immune system, especially in cell-mediated immunity. This could explain a significantly higher frequency of autoimmune disorders in DS compared to the general population. The diagnosis of autoimmune diseases accompanying DS could be complicated by masking effects of the underlying features of the syndrome such as failure to thrive, short stature and delayed puberty. However, screening for immunological signs of coexistent autoimmunity such as tissuespecific antibodies and monitoring insulin secretion, glucose levels, thyroid function and other metabolic parameters should help in early diagnosis of coexistent autoimmunity in DS patiens.
\end{abstract}

Keywords: autoimmune thyroid disease - celiac disease - coexistence - Down syndrome - type 1 diabetes

\section{IMMUNE FUNCTION IN DOWN SYNDROME}

Down syndrome (DS) is one of the most common survivable chromosomal disorders, which occurs with an incidence of about 1 per 1,000 live births. Down syndrome is well established to be associated with multiple immune aberrations. Compared to healthy subjects, DS patients are significantly more sensitive to infections and have

Dimitry Chistiakov, Department of Pathology, University of Pittsburgh Medical Center, A719 Scaife Hall, 3550 Terrace st., Pittsburgh, PA15261, USA

ㅁac58@pitt.edu

ㅇㅛㅛ +14128026114

且 +14128026799 higher frequency of coexistent hepatitis B and malignant disease (especially leukemia) in addition to the enhanced predisposition to autoimmune disease (Nespoli et al. 1993).

The DS thymus displays pathological changes in morphology, with diminished cortex and enlarged Hassal's corpuscles (Larocca et al. 1990). A biased CD4+/CD8+ lymphocyte ratio was observed, with depletion of helper/inducer CD4+ cells and increased numbers of suppressor/cytotoxic (CD8+) cells (Tamiolakis et al. 2003). In DS patients, abnormalities in maturation and function of thymic immunoregulatory $\mathrm{T}$ cells subsets have been also reported (Philipp et al. 1986). The number of circulating natural killer cells with low activity was shown to be increased (Maccario et al. 1984). However, the significance of this observation is not clear, and it may simply reflect the altered maturation of immune cells, which is a feature of the syndrome (Nespoli et al. 1993). 
In lymphoid cells derived from patients with DS, lymphocyte-function-associated antigen-1 (LFA-1) is overexpressed (Murphy et al. 1993). This adhesion receptor plays a crucial role in the trafficking and activation of leukocytes. Accordingly, cells with trisomy 21 have increased sensitivity to interferons (INFs) (Murphy et al. 1992). The enhanced vulnerability to INF- $\gamma$, the most potent interferon in the immune system produced by activated $\mathrm{T}$ cells, may cause increased expression of the Major Histocompatibility Complex class II and ICAM-1, which is the natural ligand for LFA-1 (Murphy et al. 1992). This could be associated with enhanced presentation of an autoantigen to immune cells and hence be one of the earliest events in the development of autoimmune disease. In DS patients, depressed production of cytokines such as interleukin-2 and others, and B cell abnormalities accompanied with the aberrant phagocyte trafficking and abnormal production of oxygen radicals may also account for the predisposition to autoimmune disorders (Mahmoud et al. 2005).

In DS, the autoimmune diseases may affect both the endocrine system (thyroid, pancreatic islets and adrenal gland) (Goldacre et al. 2004) and non-endocrine tissue [gastric parietal cells (Zori et al. 1990) and intestinal mucosa (Jansson and Johansson 1995)]. Coexistent infammatory immune-related disorders such as alopecia areata (Barankin and Guenther 2001), chronic autoimmune hepatitis (McCulloch et al. 1982) and primary sclerosing cholangitis (Mehta et al. 1995) have also been observed in DS subjects. Selfantibodies are frequently present in DS and serve as markers of autoimmunity. Impaired function of the immune system results in higher vulnerability of DS patients to infections, the most common of which, oral candidiasis with clinically apparent erythematous or pseudomembranous lesions, is significantly more prevalent in children with Down syndrome $(40 \%)$ compared to the normal controls $(<1 \%)$ (Carlstedt et al. 1996).

Feingold et al. (1990) suggested that some abnormalities of Down syndrome could be attributed to the overexpression at specific loci, resulting from the presence of two identical copies of a susceptibility allele inherited from the parent of origin of trisomy (disomic homozygosity). By inheriting two identical copies of a rare susceptibility allele, the individual with Down syndrome exceeds a liability threshold and manifests the disorder. In many respects, DSassociated autoimmune diseases are similar to those seen in autoimmune polyglandular syndrome type 1 , which is mapped to chromosome 21q22.3. Shield et al. (1999) hypothesized that autoimmune manifestations in DS could be related to the disomic homozygosity in this region. However, this hypothesis was rejected when no increased disomic homozygosity have been found on 21q22 in DS patients affected with type 1 diabetes (T1D) compared to non-complicated DS subjects (Shield et al. 1999).

\section{AUTOIMMUNE THYROID DISEASE AND DOWN SYNDROME}

Autoimmune thyroid disease (AITD) is the most frequent autoimmune disorder coexisting with DS. Among two major clinical outcomes of AITD, Graves' disease and Hashimoto's thyroiditis (HT), HT most often accompanies DS (Kinik et al. 2006). Histologically, HT is characterized by lymphocytic infiltration of the thyroid. $\mathrm{T}$ cells and $\mathrm{B}$ cells account for about $60 \%$ and $30 \%$ of infiltrating lymphocytes, respectively. The $\mathrm{T}$ cell population is mostly represented by helper (CD4+) and cytotoxic (CD8+) cells (Lorini et al. 2003).

The onset of the disorder is usually insidious. Most patients have a euthyroid goiter or a goiter with mild hypothyroidism, and sometimes the child exhibits hypothyroidism with growth retardation. A few children have clinical symptoms suggestive for hyperthyroidism such as nervousness, irritability, increased sweating or hyperactivity. The thyroid gland is usually diffusely enlarged and firm with accentuation of the normal lobular architecture. Usually, the gland undergoes progressive atrophy, and the patient has acquired hypothyroidism.

Most of affected patients display detectable circulating thyroid-specific autoantibodies (Kraiem 1998). Of those, anti-thyroid peroxidase and antithyroglobulin self-antibodies are the most prevalent and hence useful for diagnosis. Blood from HT patients contains a subset of thymus-dependent Tautoreactive lymphocytes sensitized against the microsomes and cell membranes of thyroid cells (Weetman 2000).

In DS adolescents, the reported prevalence of acquired hypothyroidism, due to autoimmune thyroiditis, varies from 3 to $28 \%$ (Sare et al. 1978, Kinik et al. 2006). Children with DS also exhibit increased risk of autoimmune thyroiditis (Pueschel and Pezzullo 1985, Sharav et al. 1988, Zori et al. 1990, Ivarsson et al. 1997). Rubello et al. (1995) observed a higher prevalence of subclinical hypothyroidism and antithyroid antibodies (ATA) in DS patients compared to control subjects. Longterm (range 2-7 years, mean 3.1 years) follow-up showed that ATA-positive DS patients with subclinical hypothyroidism had a high tendency to develop clinical AITD in comparison with ATAnegative DS subjects with subclinical hypothyroidism (Rubello et al. 1995).

The frequency of ATA among patients with DS and hypothyroidism increases with age. ATA associated with hypothyroidism are significantly 
less common in DS children under 8-10 years of age. However, some studies reported ATA-positive DS children under 3 years of age (Ivarsson et al. 1997, Karlsson et al. 1998).

In blood of pregnant women with DS, elevated levels of chorionic gonadotropin (HCG) were found, while serum concentrations of alphafetoprotein ( $\alpha$-FP) were decreased (Cuckle 2000). HCG is structurally similar to thyroid-stimulating hormone (TSH) and may be important for thyroid regulation in normal pregnancy (Kennedy and Darne 1991). At higher circulating concentrations, HCG may bind to thyroid cells, thereby modulating their function and contributing to maternal and fetal thyroid abnormalities. $\alpha-\mathrm{FP}$, an acute phase reactant, plays a potentially important immunomodulatory role through inhibiting the expression of class II antigens by antigenpresenting cells (Lu et al. 1984). Therefore, changes in $\alpha$-FP secretion in DS pregnancies may be involved in the autoimmunity process.

\section{DIABETES MELLITUS AND DOWN SYNDROME}

The basic cause of the clinical diagnosis in T1D is a sharply diminished secretion of insulin. Failure of pancreatic $\beta$ cell function in T1D resulted from the autoimmune destruction of pancreatic islets in genetically predisposed individuals (Bertera et al. 1999). Evidence for isletspecific autoimmunity comes from histologic studies of the pancreas from T1D patients showing the lymphocytic infiltration around the pancreatic islets. Later, the islets become progressively hyalinized and scarred, a process suggesting for an ongoing inflammatory response.

A high percentage of newly diagnosed patients with T1D exhibits circulating autoantibodies directed against cell surface or cytoplasmic targets in islet $\beta$ cells. Autoantibodies to islet-specific antigens such as glutamic acid decarboxylase islet isoform, insulin and protein tyrosine phosphataselike antigens IA-2 and IA-2b are the most common and serve as immunologic markers of anti-islet autoimmunity (Knip 1998).

T1D occurs in $1.4-10.6 \%$ of individuals with DS that is significantly higher than in general population (0.1\%) (Milunski and Neurath 1968, VanGoor et al. 1997, Anwar et al. 1998). Furthermore, age at diabetes onset in DS infants is earlier compared with non-trisomic children (i.e., 7-8 vs. 10-14 years) (Burch and Milunsky 1969, Shield et al. 1999). In addition, a portion of children with T1D diagnosed within the first 2 years of life is markedly higher among DS population $(22 \%)$ compared to that in general population (7\%) (Shield et al. 1999).
Due to the immune dysregulation, the early onset of T1D in DS patients could suggest for a very overt autoimmune pathology compared to non-DS T1D subjects. Alternatively, the $\beta$ cell population in DS patients is more susceptible to destruction mediated by immune cells. Several studies observed a much higher prevalence of thyroid disorders in children with DS and T1D than that usually described in DS without diabetes (Griffin et al. 1997, Shield et al. 1999). This therefore suggests that the subgroup of trisomic patients with T1D carries an even higher risk of aggressive autoimmune disease.

The goals of management of T1D children with/without DS are to provide adequate nutrition and exogenous insulin in a manner that prevents polydipsia and polyuria including nocturia and weight loss, avoids ketoacidosis and severe hypoglycemia, and permits normal growth and development with an active life pattern. On the basis of the compelling evidence that the long-term complications of diabetes are related to the degree of metabolic control (DCCT Research Group, 1993, 1994), the management should aim for as nearly to the normal metabolism as possible.

According to The Diabetes Control and Complications Trial (DCCT) Group suggestions (DCCT Research Group, 1993, 1994), a nearnormal glycemic control can be attained in individuals including adolescents, who are highly motivated and willing to administer three or more insulin injections based on the results of multiple daily home blood glucose monitoring measurements or who can effectively use a programmable pump to deliver insulin. This intensive treatment should be accompanied by close attention to the nutritional intake and exercise. Thus, to achieve the treatment goals, patients must understand the principles of the pathophysiology and management of the disease. However, in DS patients, this may be difficult.

To overcome this problem, Anwar et al. (1998) treated patients with DS and diabetes with a oncedaily administration of insulin (a regimen often associated with suboptimal glycemic control). The authors found that the mean $\mathrm{HbAlc}$ value of more than a half study group was similar to that of a control group of diabetic patients matched for age, sex and disease duration and treated with multiple daily injections of insulin. Anwar et al. (1998) attributed these results to the more stable lifestyle of the patients with DS, which may assist in the maintenance of stable glycemic control, particularly with respect to diet and compliance. The provision of fewer daily insulin injections can be helpful in individuals with variable levels of disability who are dependent on others for their medication and care.

In diabetic patients with DS, a systolic blood pressure and plasma cholesterol were significantly 
lower compared with the matched non-DS diabetic controls (Anwar et al. 1998). A low prevalence of diabetic retinopathy in DS patients with long-term diabetes (mean 17.6 years) was also found suggesting for some inherent protective factor against the development of microvascular diabetic complications and atherosclerosis. (Fulcher et al. 1998).

\section{CELIAC DISEASE AND DOWN SYNDROME}

Celiac disease (CD) is an autoimmune gastrointestinal disorder characterized by mucosal atrophy of the jejunum on exposure to gluten and related proteins from certain cereals. The immunological response to gluten results in injury to the small bowel, with villous atrophy, crypt hyperplasia and damage to the surface epithelium. The clinical presentation is variable, with different degrees of malabsorption manifested as diarrhea, anorexia with failure to gain weight, abdominal pain and abdominal distention, anemia, failure to thrive (Visakorpi and Maki 1994). CD is accompanied by mucosal $\operatorname{IgA}$ autoantibodies to reticulin, endomysium and transglutaminase (Chan et al. 1994, Dietrich et al. 1997). Screening based on $\operatorname{IgA}$ endomysial autoantibodies provides an almost $100 \%$ sensitivity and specificity (Corrao et al. 1994).

The standard for confirmation of CD diagnosis is the histologic study of small bowel biopsy. Biopsy should be performed in the presence of a high suspicion of $\mathrm{CD}$ or findings of serum endomysial antibody. Treatment consists of a lifelong, strict gluten-free diet.

CD frequently coexists with DS affecting 743\% DS patients (Zubillaga et al. 1993, Jansson and Johansson 1995, Carnicer et al. 2001). This frequency is significantly higher than $\mathrm{CD}$ incidence $(0.5 \%)$ in general population. The pathogenic factor that links both diseases may be an altered immune system or the presence of a common genetic factor.

DS children with/without CD cannot be distinguished by clinical and growth characteristics. Book et al. (2001) reported similar rates of diarrhea, constipation, vomiting and abdominal pain for these two groups. Only bloating symptoms were significantly more frequent in those with CD. Therefore, determinations of antigliadin and antiendomysium antibodies should be included in the routine follow-up of patients with DS. Intestinal biopsy should be performed when antibody values are above the cut-off. Otherwise, the diagnosis of $\mathrm{CD}$ can be considerably delayed.

\section{CONCLUSION}

DS coexists with several immune-related disorders the most frequent of which are HT, T1D and CD. Of coexistent autoimmune disorders, autoimmune thyroiditis is the most common. The diagnosis of autoimmune diseases accompanying DS could be complicated by masking effects of the underlying features of the syndrome such as failure to thrive, short stature and delayed puberty. Physicians may erroneously attribute DS symptoms to a new disease because of the difficulty to obtain a clear history due to the patients' limited expressive speech and their low tendency to complain of discomfort. A high index of suspicion is needed in order to identify those patients with concomitant immune-related disorders. Therefore, follow-up of growth patterns, searching clinical signs of diabetes, thyroid function monitoring, screening for thyroid- and islet-specific autoantibodies and measurement of serum concentrations of antigliadin and antiendomysium self-antibodies should be started from infancy to prevent further deterioration of growth and mental development.

\section{REFERENCES}

Anwar AJ, Walker JD, Frier BM: Type 1 diabetes mellitus and Down's syndrome: prevalence, management and diabetic complications. Diabet. Med. 15:160-163, 1998.

Barankin B, Guenther L: Dermatological manifestations of Down's syndrome. J. Cutan. Med. Surg. 5:289-293, 2001.

Bertera S, Alexander A, Giannoukakis N, Robbins PD, Trucco M: Immunology of type 1 diabetes. Endocrinol. Metab. Clin. North Am. 28:841-846, 1999.

Book L, Hart A, Black J: Prevalence and clinical characteristics of celiac disease in Down's syndrome in a US study. Am. J. Med. Genet. 98:70-74, 2001.

Burch PRJ, Milunsky A: Early onset diabetes mellitus in the general and Down's syndrome populations. Lancet 1:554-558, 1969.

Carlstedt K, Krekmanova L, Dahllöf, G., Ericsson, B., Braathen, G., Modéer, T.: Oral carriage of Candida species in children and adolescents with Down's syndrome. Int. J. Paediatr. Dent. 6:95-100, 1996.

Carnicer J, Farre C, Varea V, Vilar P, Moreno J, Artigas J: Prevalence of coeliac disease in Down's syndrome. Eur. J. Gastroenterol. Hepatol. 13:263-267, 2001. 
Chan KN, Phillips AD, Mirakian R, Walker-Smith JA: Endomysial antibody screening in children. J. Pediatr. Gastroenterol. Nutr. 18:316-320, 1994.

Corrao G, Corazzo GR, Andreani ML, Torchio P, Valentini RA, Galatola G, Quaglino D, Gasbarrini G, Diorio F: Serological screening of celiac disease: Choosing the optimal procedure according to various prevalence values. Gut 35:771-775, 1994.

Cuckle H: Biochemical screening for Down syndrome. Eur. J. Obstet. Gynecol. Reprod. Biol. 92:97-101, 2000.

DCCT Research Group:: The effect of intensive treatment of diabetes on the development and progression of long-term complications in insulin-dependent diabetes mellitus. N. Engl. J. Med. 329:977-986, 1993.

DCCT Research Group: Effect of intensive diabetes treatment on the development and progression of long-term complications in adolescents with insulin-dependent diabetes mellitus: Diabetes Control and Complications Trial. J. Pediatr. 125:177-188, 1994.

Dietrich W, Ehnis, Bauer M, Donner P, Volta U, Riecken EO, Schuppan D: Identification of tissue transglutaminase as the autoantigen of celiac disease. Nat. Med. 3:797-801, 1997.

Feingold E, Lamb NE, Sherman SL: Methods for genetic linkage analysis using trisomies. Am. J. Hum. Genet. 56:475-483, 1990.

Fulcher T, Griffin M, Crowley S, Firth R, Acheson $\mathrm{R}, \mathrm{O}^{\prime}$ Meara N: Diabetic retinopathy in Down's syndrome. Br. J. Ophthalmol. 82:407-409, 1998.

Goldacre MJ, Wotton CJ, Seagroatt V, Yeates D: Cancers and immune related diseases associated with Down's syndrome: a record linkage study. Arch. Dis. Child. 89:10141017, 2004.

Griffin ME, Fulcher T, Nikookam K, Crowley J, Firth RG, OMeara NM. Down's syndrome, IDDM, and hypothyroidism. Diabetes Care 20: 1202-1203, 1997.

Ivarsson SA, Ericsson VB, Gustafsson J, Forslund M, Forslund M, Vegfors P, Anneren G: The impact of thyroid autoimmunity in children and adolescents with Down syndrome. Acta Pediatr. 86:1065-1067, 1997.

Jansson U, Johansson C: Down syndrome and celiac disease. J. Pediatr. Gastroenterol. Nutrition 21:443 - 445, 1995.

Karlsson B, Gustafsson J, Hedov G, Ivarsson SA: Thyroid dysfunction in Down's syndrome: relation to age and thyroid autoimmunity. Arch. Dis. Child. 79:242-245, 1998.

Kennedy RL, Darne J: The role of hCG in regulation of the thyroid gland in normal and abnormal pregnancy. Obstet. Gynecol. 78:298307, 1991.
Kinik ST, Ozcay F, Varan B: Type I diabetes mellitus, Hashimoto's thyroiditis and celiac disease in an adolescent with Down syndrome. Pediatr. Int. 48:433 - 435, 2006.

Knip M: Prediction and prevention of type 1 diabetes. Acta Paediatr. Suppl. 425:54-62, 1998.

Kraiem Z: The measurement of antithyroid autoantibodies in the diagnosis and management of thyroid autoimmune disease. Clin. Rev. Allergy Immunol. 16:219-225, 1998.

Larocca LM, Lauriola L, Ranelleti FO, Piantelli M, Maggiano N, Ricci R, Capelli A: Morphological and immunohistochemical study of Down syndrome thymus. Am. J. Med. Genet. Suppl. 7:225-230, 1990.

Lorini R, Gastaldi R, Traggiai C, Perucchin PP: Hashimoto's thyroiditis. Pediatr. Endocrinol. Rev. 2:205-211, 2003.

Lu CY, Changelian PS, Unanue ER: Alphafetoprotein inhibits macrophage expression of Ia antigens. J. Immunol.132:1324-1327, 1984.

Maccario R, Ugazio AG, Nespoli L, Alberini C, Montagna D, Porta F, Bonetti F, Burgio GR: Lymphocyte subpopulations in Down's syndrome: high percentage of circulating HNK-1+, lue2a + cells. Clin. Exp. Immunol. 57:220-226, 1984.

Mahmoud SA, Lowery-Nordberg M, Chen H, Thurmon $\mathrm{T}$, Ursin S, Bahna SL: Immune defects in subjects with dysmorphic disorders. Allergy Asthma Proc. 26:373-381, 2005.

McCulloch AJ, Ince PG, Kendall-Taylor P: Autoimmune chronic active hepatitis in Down's syndrome. J. Med. Genet. 19:232-234, 1982.

Mehta DI, Hill ID, Singer-Granick C et al.: Primary sclerosing cholangitis and multiple autoimmune disorders in a patient with Down syndrome. Clin. Pediatr. (Phila) 34:502-505, 1995.

Milunski A, Neurath PW: Diabetes mellitus in Down's syndrome. Arch. Environ. Health 17:372-376, 1968.

Murphy M, Hyun W, Hunte B, Levine A et al.: A role for tumor necrosis factor-alpha and interferon-gamma in the regulation of interleukin-4-induced human thymocyte proliferation in vitro. Heightened sensitivity in the Down syndrome (trisomy 21) thymus. Pediatr. Res. 32:269-276, 1992.

Murphy M, Insoft RM, Pike-Nobile L et al.: Overexpression of LFA-1 and ICAM-1 in Down syndrome thymus. Implications for abnormal thymocyte maturation. J. Immunol. 150:5696-5703, 1993.

Nespoli L, Burgio GR, Ugazio AG, Maccario R: Immunological features of Down syndrome: a 
review. J. Intellect. Disabil. Res. 37:543-551, 1993.

Philip R, Berger AC, McManus NH, Warner NH, Peacock MA, Epstein LB: Abnormalities in the in vitro cellular and humoral responses to tetanus and influenza antigens with concomitant numerical alterations in lymphocyte subsets in Down's syndrome (trisomy 21). J. Immunol. 136:1661-1667, 1986.

Pueschel SM, Pezzullo JC: Thyroid dysfunction in Down syndrome. Am. J. Dis. Child. 139:636639, 1985.

Rubello D, Pozzan GB, Casara D, Girelli ME, Boccato S, Rigon F, Baccichetti C, Piccolo M, Betterle C, Busnardo B: Natural course of subclinical hypothyroidism in Downs syndrome - prospective study results and therapeutic considerations. J. Endocrinol. Invest. 18: 35-40, 1995.

Sare Z, Ruvalcaba RHA, Kelly VC: Prevalence of thyroid disorder in Down syndrome. Clin. Genet. 14:154-158, 1978.

Sharav T, Collins RM, Baab PJ: Growth studies in infants and children with Down's syndrome and elevated levels of thyrotropin. Am. J. Dis. Child. 142:1302-1306, 1988.

Shield JPH, Wadsworth EJK, Hassold TJ, Judis LA, Jacobs PA: Is disomic homozygosity at the APECED locus the cause of increased autoimmunity in Down's syndrome? Arch. Dis. Child. 81:147-150, 1999.

Tamiolakis D, Venizelos I, Kotini A, Nikolaidou S, Papadopoulos N: Prevalence of CD8/CD4 ratio in the fetal thymic parenchyme in Down's syndrome. Acta Medica (Hradec Kralove) 46:179-182, 2003.

VanGoor JC, Massa GG, Hirasing R: Increased incidence and prevalence of diabetes mellitus in Down's syndrome. Arch. Dis. Child. 77:186, 1997.

Visakorpi J, Maki M: Changing clinical features of coeliac disease. Acta Paediatr. Suppl. 395:1013, 1994.

Weetman AP: Chronic autoimmune thyroiditis. In Braverman LE, Utiger RD (eds.): The Thyroid, A Fundamental and Clinical Text, $8^{\text {th }}$ ed., Lippincott Williams and Wilkins, Philadelphia 2000, pp. 721-732.

Zori RT, Schatz DA, Ostrer H, Williams CA, Spillar R, Riley WJ: Relationship of autoimmunity to thyroid dysfunction in children and adults with Down syndrome. Am. J. Med. Genet. Suppl. 7:238-241, 1990.

Zubillaga P, Vitoria JC, Arrieta A, Echaniz P, Garciamasdevall MD.: Down's syndrome and celiac disease. J. Pediatr. Gastroenterol. Nutr. 16:168-171, 1993. 